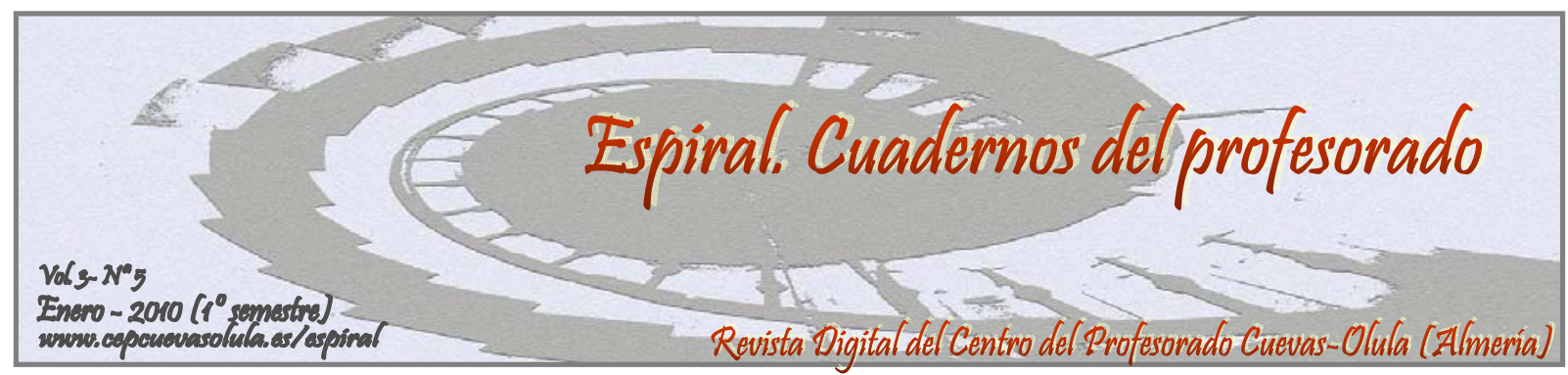

\title{
EFECTOS DE LA INGESTA DE DIFERENTES CONCENTRACIONES DE CARBOHIDRATOS EN EL RENDIMIENTO EN CICLOERGÓMETRO: ESTUDIO PRELIMINAR
}

\section{EFFECTS OF INTAKE OF DIFFERENT CONCENTRATIONS OF CARBOHYDRATE ON PERFORMANCE CYCLE ERGOMETER: PRELIMINARY STUDY.}

\section{María del Pilar Vílchez Conesa ${ }^{1}$ y Dr. Manuel Gómez López ${ }^{2}$}

(1) Doctoranda. Departamento Actividad Física y Deporte. Universidad de Murcia.

${ }^{(2)}$ Facultad de Ciencias del Deporte. Universidad de Murcia

RESUMEN: De acuerdo al papel de los carbohidratos en el ejercicio físico prolongado e intenso, se investigan los efectos en la ingesta de soluciones de baja y alta concentración en carbohidratos comprobando si pueden proveer de más energía para mejorar el rendimiento. Se utilizaron, a doble ciego, tres tipos de soluciones en las mismas condiciones: placebo, baja y alta concentración de carbohidratos en tres sujetos con una prueba de intensidad máxima de 45 minutos en cicloergómetro. Los resultados mostraron que la suplementación de carbohidratos no mejora el rendimiento en un ejercicio durante 45 minutos de intensidad moderada-alta en cicloergómetro. No se encontraron cambios en el rendimiento durante los primeros diez minutos de ejercicio intenso, aunque sí se encontraron mejoras en algunas variables que influyen en el rendimiento, como el ritmo cardiaco, el mantenimiento de altos niveles de glucosa en sangre, una menor producción de lactato y una menor percepción del esfuerzo durante la actividad.

Palabras clave: carbohidratos, rendimiento, soluciones energéticas.

ABSTRACT: According to the role of carbohydrates in the prolonged and intense exercise, we investigate the effects on the intake of solutions of low and high concentration of carb checking if they can provide more energy to improve performance. We used double-blind, three types of solutions under the same conditions: placebo, low and high concentration of carbohydrates in three subjects with a test of 45 minutes maximum intensity cycle ergometer. The results showed that carbohydrate supplementation does not improve exercise performance during 45 minutes of moderate-high intensity cycle ergometer. There were no changes in performance during the first ten minutes of intense exercise, although there were improvements in some variables affecting performance, such as heart rate, maintenance of high blood glucose levels, reduced lactate production and a lower perceived effort during activity.

Key words: carbohydrates, performance, energy solutions.

Vílchez, M.P. \& Gómez, M. (2010). Efectos de la ingesta de diferentes concentraciones de carbohidratos en el rendimiento en cicloergómetro: estudio preliminar. Espiral. Cuadernos del Profesorado [en línea], 3(5), 51-58. Disponible en: http://www.cepcuevasolula.es/espiral. 


\section{1.- INTRODUCCIÓN.}

Los CHO aportan la energía necesaria para la contracción muscular (Shirreffs, 1998), por eso son una fuente de energía muy recomendada durante el ejercicio (Petrie, Stover y Horswill, 2004). De hecho, la fatiga está estrechamente relacionada con la depleción del almacén de CHO en el músculo y en el hígado (Williams, 2004) y una suplementación de CHO retrasa la fatiga durante los 30-60 minutos de ejercicio (Coyle, 1999).

Haman y cols. (2004) hablan de una flexibilidad en la utilización de vías metabólicas para la obtención de energía. Sin embargo, no hay evidencia científica de la mejora del rendimiento debida a una suplementación de CHO. Según Shirreffs (1998), no todos los estudios muestran un efecto positivo en el rendimiento con la suplementación, pero tampoco hay evidencias de exista un efecto adverso en el rendimiento. Los CHO son los más destacados cuando se habla de suplementación durante el ejercicio (Vitasovic y Saldanha, 2003).

$\mathrm{Si}$ la intensidad del ejercicio aumenta, también lo hace la utilización de CHO (Petrie, Stover y Horswill, 2004). La contribución relativa del glucógeno muscular y la glucosa sanguínea a la energía metabólica durante el ejercicio varía en función de la intensidad y la duración del ejercicio. La glucosa en sangre juega un mayor papel durante el ejercicio de baja intensidad, mientras el glucógeno muscular es una fuente primaria de CHO durante el ejercicio de alta intensidad (Powers y Howley, 2001). Se mantienen niveles altos de glucosa sanguínea con una ingesta de $\mathrm{CHO}$ durante el ejercicio porque los músculos que trabajan utilizan mayoritariamente esta energía consumida, particularmente en las etapas más tardías del ejercicio prolongado (Shirreffs, 1998).

Una vez revisados los estudios que apoyan la suplementación con CHO durante el ejercicio, se deben conocer las condiciones sobre la administración en cuanto a cantidad o concentración, forma de suministrarlos y frecuencia de la ingesta durante el ejercicio de acuerdo a los parámetros del presente estudio, pues Bosch y Noakes (2005) apoyan el efecto ergogénico de la ingesta de CHO pero consideran que estos parámetros todavía no han sido evidenciados.

Coyle (1999) aconseja que los CHO se consuman para suplir a la glucosa sanguínea con glucosa exógena aproximadamente en 1g/minuto, hasta el final del ejercicio. Durante el ejercicio, Powers y Howley (2001) afirman que es necesario un suplemento de CHO entre 30 y 60 gramos por hora para aumentar el rendimiento, como han utilizado la mayoría de estudios experimentales (Coyle, 1999). Nassis, Williams y Chisnall (1998) utilizaron una solución concentrada al 6.9\% de $\mathrm{CHO}$ durante ejercicio intermitente submáximo pero no encontraron un retraso de la fatiga. En general, recpecto a la concentración o cantidad de CHO, Shirreffs (1998) afirma que la contribución de los CHO al metabolismo es dependiente de la carga de trabajo (siendo mayor a intensidades altas que a bajas).

Angus et al. (2000) citado en (Vitasovic y Saldanha, 2003), realizaron un estudio con test en cicloergómetro, bebiendo un suplemento de $\mathrm{CHO}$ cada 15 minutos, y observaron que sólo disminuyó la intensidad del ejercicio en el grupo placebo. L ingesta de glucosa tiene un efecto en la utilización de glucógeno muscular durante el ejercicio prolongado en cicloergómetro a $70 \%$ a $75 \% \mathrm{VO}_{2} \max$, y es posible que la ingesta de CHO inhiba la movilización de las reservas de glucógeno hepático como afirma Shirreffs (1998).

Las bebidas de $\mathrm{CHO}$ pueden incrementar el rendimiento en ejercicios de resistencia y favorecer la eliminación del lactato retrasando la fatiga (Khanna y Manna, 2005). La ingesta de $\mathrm{CHO}$ en forma de bebida concentrada durante esfuerzos de alta intensidad ayuda a la capacidad de mantener una velocidad alta, mantener potencia de piernas y al rendimiento en las habilidades motoras tras el esfuerzo (Petrie, Stover y Horswill, 2004). Al mismo tiempo, la forma líquida de la suplementación de CHO (al $6,5 \%)$ y electrolitos permite una rehidratación para cubrir el $150 \%$ de la masa corporal perdida durante los primero 90 minutos de carrera. El rendimiento de resistencia es maximizado cuando realiza la suplementación mediante fluido y $\mathrm{CHO}$ (Coyle, 1999). En esta línea, Coyle (1986); Maughan (1991), citado en Powers y Howley (2001) afirman que la ingesta de CHO a través de soluciones deportivas pueden mejorar el rendimiento aeróbico durante el ejercicio submáximo ( $<70 \% \mathrm{VO}_{2}$ max) y de larga duración (>90minutes).

En el presente estudio preliminar se observan los efectos que tiene la ingesta de diferentes concentraciones de $\mathrm{CHO}$ en el 
rendimiento individual, durante un ejercicio de intensidad alta y duración moderada (45 minutos). Se entiende por rendimiento la mejora de la capacidad de trabajo en cicloergómetro, medida con cinco variables: el ritmo cardiaco (a menor ritmo cardiaco, posibilidad de un mejor rendimiento), nivel de glucosa (a mayor nivel de glucosa, un posible mayor sustrato energético y mejora del rendimiento), nivel de lactato en sangre (a menor nivel de lactato producido en sangre, posibilidad de mejorar el rendimiento), esfuerzo percibido (a menor esfuerzo percibido, posibilidad de aumentar la intensidad o disminuir la fatiga) y distancia total recorrida en los 45 minutos de la prueba (a mayor distancia, mayor rendimiento).

\section{2.- MÉTODO.}

\section{Diseño.}

El tipo de diseño fue un estudio de caso. El estudio fue un related test, con una variable independiente y más de dos condiciones. $\mathrm{La}$ variable independiente fue la solución de CHO. Las variables dependientes fueron el rendimiento en cicloergómetro medido mediante el seguimiento de cinco variables: el ritmo cardiaco, la glucosa en sangre, el lactado en sangre, el esfuerzo percibido y la distancia recorrida. Las condiciones fueron tres soluciones diferentes: solución placebo, solución alta en CHO, al 12\%, y otra baja en $\mathrm{CHO}$, al 6\%.

Tabla 1.- Características de los participantes del estudio.

\begin{tabular}{lcccc}
\hline & Género & Edad & Peso & Talla \\
\hline Participante 1 & Mujer & 21 & $48 \mathrm{~kg}$ & $1,60 \mathrm{~m}$ \\
Participante 2 & Mujer & 22 & $56 \mathrm{~kg}$ & $1,64 \mathrm{~m}$ \\
Participante 3 & Hombre & 22 & $65 \mathrm{~kg}$ & $1,73 \mathrm{~m}$ \\
\hline
\end{tabular}

\section{Participantes.}

Tres participantes formaron parte del estudio: un hombre y dos mujeres, estudiantes de la asignatura "Physiology of Elite" en la facultad Trinity and All Saints de la Universidad de Leeds. Los participantes eran físicamente activos y no tenían ningún problema médico (tabla 1).

\section{Procedimiento.}

Los participantes rindieron tres pruebas físicas, separadas temporalmente por una semana, excepto la última que fue separada por dos semanas. Se controló que los participantes comieran moderadamente dos o tres horas antes al rendimiento de la prueba y se les aconsejó continuar con una dieta equilibrada durante las semanas que duró la investigación. Los participantes debían seguir unos patrones de ejercicio similar el día previo a cada prueba.

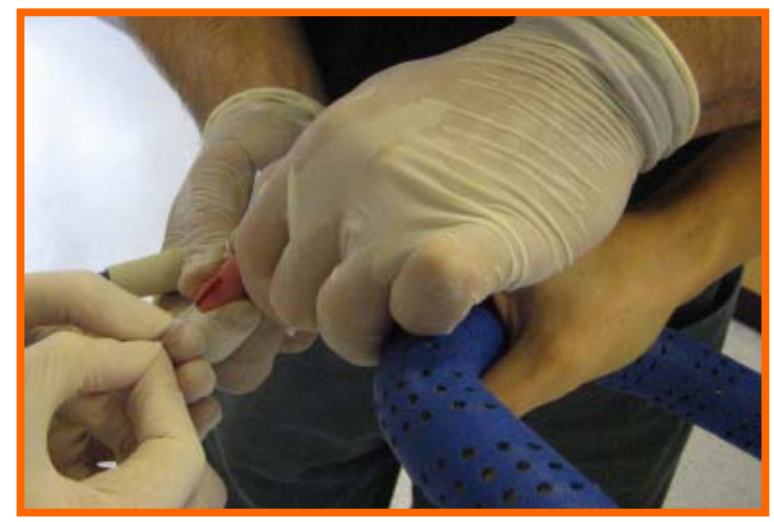

Figura 1.- Ejemplo de recogida de una muestra de sangre para determinar la glucosa.

Cada participante cumplimentó un consentimiento firmado de un cuestionario sobre la historia clínica y su acuerdo con la participación en el estudio. Además, se llevaron a cabo protocolos de seguridad requeridos para las muestras de sangre (figuras 1,2 y 3 ).

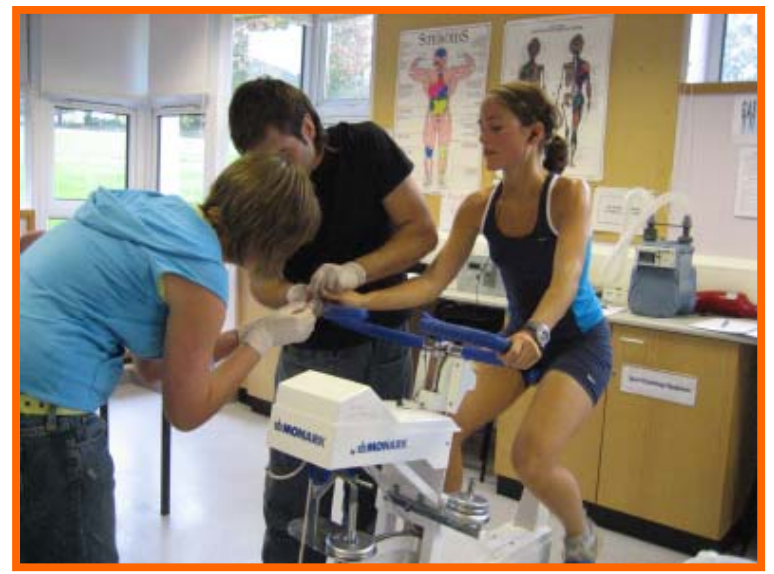

Figura 2.- Ejemplo de recogida de una muestra de sangre para determinar la glucosa durante el ejercicio.

Se utilizó un día previo para explicar al atleta el protocolo del estudio. Dicho encuentro se utilizó para obtener los datos del peso (sin zapatillas de deporte y con la ropa de la prueba), ajustarle el monitor y el pulsómetro a cada participante, y ajustar la resistencia y el manillar del ergómetro a las medidas del participante. 


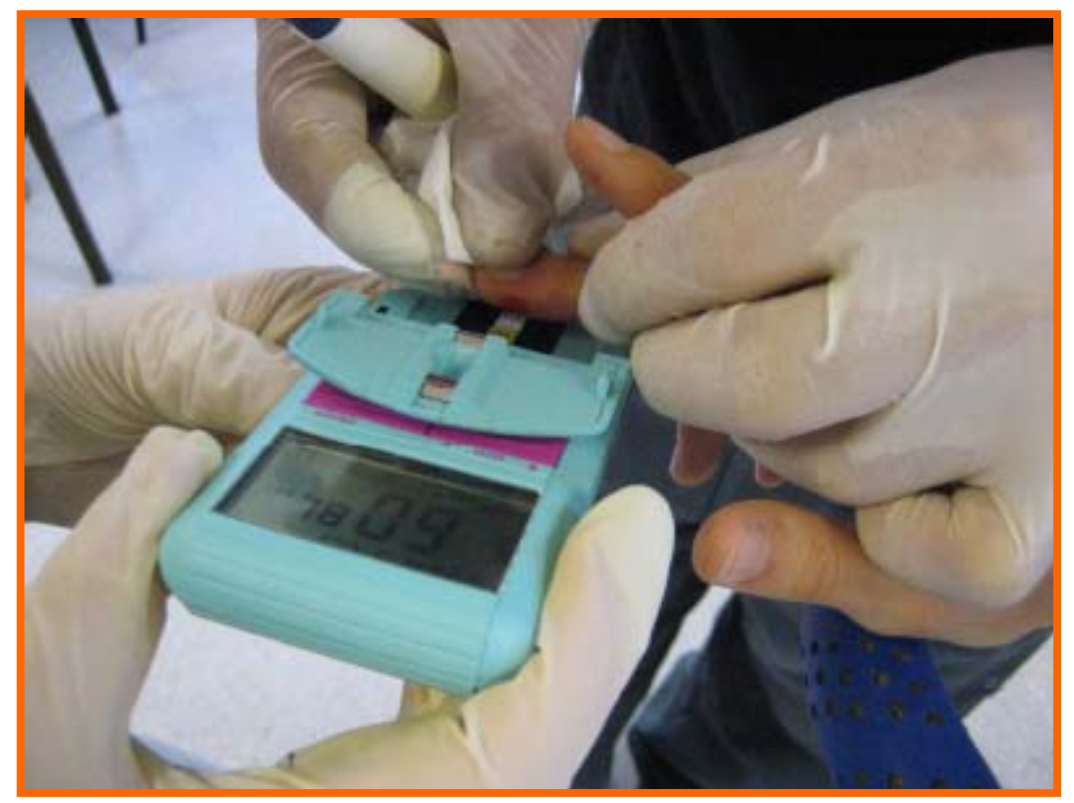

Figura 3.- Ejemplo de cómo se determinó el lactato en sangre

Cada prueba consistía en rendir 45 minutos a la máxima intensidad en el cicloergómetro de la marca y modelo "Monark 884E” (figura 4); ingiriendo una solución placebo, otra de baja y otra de alta concentración de $\mathrm{CHO}$ en cada prueba. Cada participante ingirió $5 \mathrm{ml}$ por kilogramo de peso corporal de fluido 30 minutos antes de la prueba (por ejemplo, la participante de $48 \mathrm{~kg}$, mujer, ingiere $245 \mathrm{ml} 30$ minutos antes de la prueba). Y durante la prueba, cada participante consumió $2 \mathrm{ml}$ por kilogramo de peso cada 10 minutos, por ejemplo, el participante anterior ingiere $98 \mathrm{ml}$ cada 10 minutos. Se recomendó a los participantes beber a pequeños sorbos para prevenir náuseas. Las tomas de datos se realizan justo antes de beber la solución.

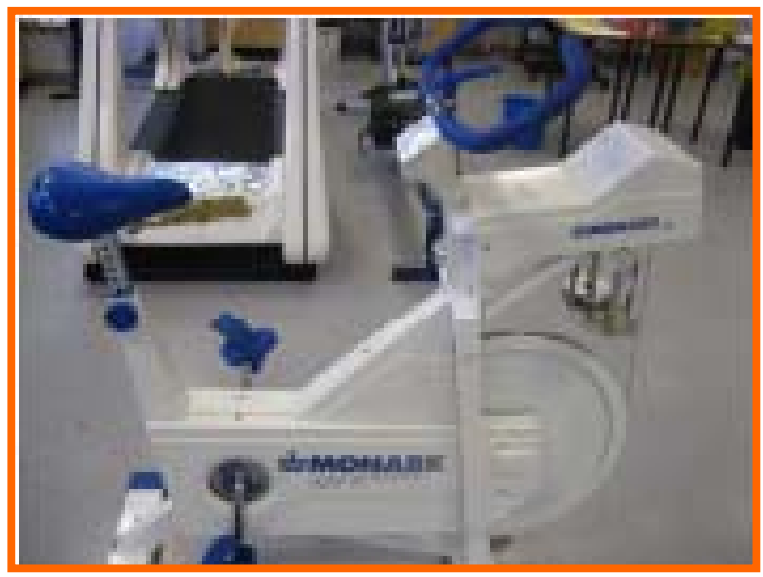

Figura 4.- Cicloergómetro Monark 884E .
Cada participante realizó 5 minutos de calentamiento a una intensidad media. Se midió la frecuencia cardiaca de reposo antes de cada prueba. Para evitar que los sujetos tuvieran una ventaja o influencia psicológica, fue un estudio de doble ciego. Las soluciones no fueron utilizadas con los detalles de su contenido pero se midieron según las características del participante (con recipientes de medida y soluciones, Figura 5). Para medir la intensidad del ejercicio fue utilizada la escala del ratio de percepción de Borg (1982) (figura 5).

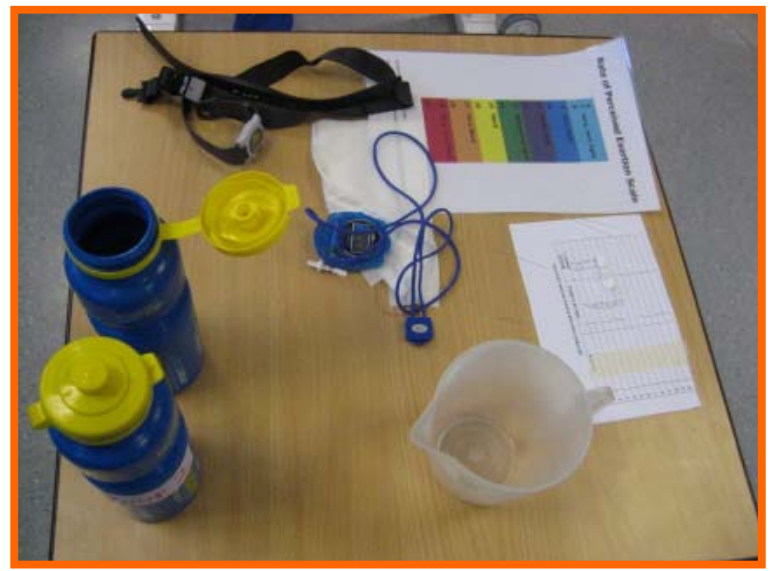

Figura 5.- Material utilizado.

Cada sujeto fue controlado por un grupo de trabajo formado por 3-4 personas. Cada grupo era responsable de controlar las variables, realizar la toma sanguínea de glucosa y lactato, preguntar al participante el esfuerzo percibido, y apuntar la distancia recorrida y la frecuencia cardiaca. La 
recogida de datos se realizó al inicio de la prueba y cada diez minutos hasta los 45 minutos.

Al final del ejercicio, en el minuto 45 , se midieron todas las variables por última vez. Se realizó una vuelta a la calma a una intensidad progresivamente menor.

\section{3.- RESULTADOS.}

En la tabla 2 se muestran los valores promedios y la desviación típica de los participantes en tres tomas: la toma inicial, a los 20 minutos y la toma final, en cada una de las variables controladas y en cada una de las pruebas realizadas: condición placebo, solución al $6 \%$ de $\mathrm{CHO}$ y al $12 \%$ de CHO.

En la frecuencia cardiaca de los sujetos en las tres condiciones de solución concentrada no existe una diferencia mayor a 30 pulsaciones en ningún intento, por lo que las diferencias en cuanto a la intensidad alcanzada son mínimas.

En el nivel de partida de glucosa en la prueba, tras la ingesta inicial 30 minutos antes de la prueba, se registra una mayor desviación típica en las soluciones carbohidratadas, siendo mayor en la solución más concentrada de CHO. Sin embargo, en el resto de tomas durante la prueba, las diferencias son muy pequeñas.

El nivel de lactato es menor con menor desviación entre los sujetos en ambas concentraciones de $\mathrm{CHO}$, encontrando diferencias menores entre los sujetos en las tomas registradas durante y al final de las pruebas.

Se encuentran mayores desviaciones entre los sujetos en el esfuerzo percibido de los participantes cuando se ingieren las soluciones de CHO.

También se registran mayores desviaciones en la distancia recorrida en ambas soluciones de $\mathrm{CHO}$, encontrando mayores diferencias en la solución concentrada al 6\% de CHO.

En la tabla 3 se muestra la diferencia entre el promedio los registros de los participantes en ambas condiciones de solución concentrada de CHO con la situación placebo, en tres tomas durante la prueba: toma inicial, a los 20 minutos $\mathrm{y}$ final.

Existe una mayor disminución de la frecuencia cardiaca en el minuto 20 en ambas concentraciones de $\mathrm{CHO}$, sin mostrar prácticamente diferencias en la concentración de $\mathrm{CHO}$ en la frecuencia cardiaca en el resto de las tomas.
Excepto en el minuto 20 de la concentración al $12 \%$ de $\mathrm{CHO}$ existe una pequeña mayor concentración de glucosa en los sujetos respecto a la situación placebo.

A excepción de la toma final de la concentración al $12 \%$ de $\mathrm{CHO}$, se muestra una disminución del lactato en todas las tomas en ambas concentraciones de solución carbohidratada.

No existen casi diferencias entre el esfuerzo percibido al ingerir ninguna solución con respecto a la situación placebo. Las diferencias entre la distancia recorrida en las tres situaciones posibles también es mínima.

\section{4.- DISCUSIÓN.}

No se encontraron diferencias significativas en el total de la distancia recorrida (ver tabla 2), de hecho, la significancia del total de distancia recorrida en las pruebas con soluciones placebo fueron mayores que en las pruebas de soluciones de baja concentración de CHO. Los resultados mostraron que la ingesta de $\mathrm{CHO}$ no mejora el rendimiento en el ejercicio de 45 minutos de intensidad máxima en cicloergómetro.

Aunque los participantes fueron avisados para que el ejercicio fuera de intensidad máxima, la media del ritmo cardiaco es menor de la máxima, indicando una intensidad moderada. Sin embargo, puede ser posible que la ingesta de CHO influencie positivamente en el ritmo cardiaco, pero no hay literatura al respecto. Se muestra que una solución concentrada al $12 \%$ de CHO mantiene menor número de pulsaciones por minuto en los primeros diez minutos de ejercicio.

Los resultados de glucosa pueden ser menores en una ingesta de baja concentración de $\mathrm{CHO}$ que con soluciones placebo, refiriéndose esto a que la ingesta de CHO no incrementa la circulación de glucosa en la sangre que podría ser interesante en el rendimiento como muestra la revisión de la literatura, pero no sucede en los diez primeros minutos de ejercicio máximo, que es mejorado con soluciones de una baja concentración de CHO. Esto podría indicar que la concentración de una solución de CHO podría influir más positivamente al $6 \%$ mejor que al $12 \%$ si la importancia del rendimiento dependiera en gran medida de los diez primeros minutos de la prueba. Entre participantes hubo diferencias significativas pero no las hubo entre intentos o pruebas, esto puede indicar que la ingesta de 
Efectos de la ingesta de diferentes concentraciones de carbohidratos en

el rendimiento en cicloergómetro: estudio preliminar.

Tabla 2.- Valores promedios y desviación típica de los participantes en tres tomas de cada variable en las tres condiciones de solución ingerida.

\begin{tabular}{|c|c|c|c|c|c|c|}
\hline & $\begin{array}{l}\text { Número } \\
\text { de toma }\end{array}$ & $\begin{array}{c}\text { Frecuencia } \\
\text { cardiaca }\end{array}$ & Glucosa & Lactato & $\begin{array}{c}\text { Esfuerzo } \\
\text { percibido }\end{array}$ & $\begin{array}{l}\text { Distancia } \\
\text { recorrida }\end{array}$ \\
\hline \multirow{3}{*}{ Placebo } & 0 min & $82.33 \pm 11.59$ & $5.26 \pm 0.28$ & $1.96 \pm 0.73$ & $0.00 \pm 0.00$ & $0.00 \pm 0.00$ \\
\hline & $20 \mathrm{~min}$ & $142.00 \pm 14.93$ & $3.36 \pm 0.30$ & $4.23 \pm 1.46$ & $13.00 \pm 1.00$ & $10.62 \pm 0.17$ \\
\hline & $45 \mathrm{~min}$ & $149.00 \pm 30.20$ & $4.33 \pm 0.66$ & $4.66 \pm 2.83$ & $16.66 \pm 1.15$ & $24.74 \pm 0.36$ \\
\hline \multirow{3}{*}{ CHO 6\% } & $0 \mathrm{~min}$ & $77.66 \pm 7.50$ & $5.76 \pm 1.78$ & $1.76 \pm 0.90$ & $0.00 \pm 0.00$ & $0.00 \pm 0.00$ \\
\hline & $20 \mathrm{~min}$ & $132 \pm 11$ & $4.00 \pm 0.52$ & $2.56 \pm 1.01$ & $13.33 \pm 1.53$ & $10.82 \pm 1.53$ \\
\hline & $45 \mathrm{~min}$ & $147 \pm 9.54$ & $4.36 \pm 0.50$ & $2.86 \pm 1.01$ & $16.33 \pm 2.08$ & $24.88 \pm 2.52$ \\
\hline \multirow{3}{*}{ CHO 12\% } & 0 min & $73.33 \pm 9.51$ & $6.13 \pm 2.19$ & $1.93 \pm 0.20$ & $0.00 \pm 0.00$ & $0.00 \pm 0.00$ \\
\hline & $20 \mathrm{~min}$ & $138.66 \pm 24.33$ & $3.23 \pm 0.38$ & $3.66 \pm 1.33$ & $12.66 \pm 2.08$ & $10.50 \pm 0.52$ \\
\hline & $45 \mathrm{~min}$ & $151.33 \pm 19.13$ & $4.40 \pm 0.91$ & $6.40 \pm 0.72$ & $16.66 \pm 1.53$ & $24.70 \pm 1.71$ \\
\hline
\end{tabular}

CHO durante el ejercicio depende también de las características del deportista.

En el test comparando intrasujetos hubieron diferencias significativas utilizando soluciones de baja concentración de $\mathrm{CHO}$ en la variable del lactato comparado con la solución placebo $(\mathrm{p}=0.022)$; una vez más, podría ser más interesante una concentración de $\mathrm{CHO}$ al $6 \%$ que más alta, dependiendo de los objetivos del rendimiento y de las características del deportista.

Entre participantes, hubo diferencias significativas en la variable del lactato $(p=0.002)$ dependiendo de las características individuales del deportista; una solución de CHO podría ser interesante para producir niveles más bajos de lactato durante el ejercicio.

El esfuerzo percibido de los participantes es importante también en el rendimiento deportivo, variable donde se encontraron diferencias significativas entre la ingesta de solución placebo y la solución baja en $\mathrm{CHO}(\mathrm{p}=0.043)$, y una vez más la solución baja en $\mathrm{CHO}$ parece mejorar el rendimiento y podría ser interesante en este tipo de pruebas. Sobre todo, hubieron diferencias significativas en los diez primeros minutos de la prueba en la percepción del esfuerzo del deportista $(p=0.003)$ que podría influir en la motivación y en el retraso de la fatiga y el decaimiento en los diez primeros minutos al comenzar la prueba. También se encontraron diferencias significativas en esta misma variable entre participantes $(\mathrm{p}=0.001)$, donde las características del deportista determina el rendimiento.

Las limitaciones de este estudio fueron determinadas por variables extrañas que han intentado ser controladas. Una de las más importantes es la falta de concentración de los participantes cuando tenía lugar la muestra de sangre, el participante tenía que intentar concentrarse en realizar el ejercicio a la máxima intensidad posible pero afirmaron encontrarse en una situación difícil para ello. Otra limitación podría ser la experiencia de cada participante en el cicloergómetro, pues todos eran deportistas pero ninguno entrenaba en esta especialidad deportiva. Por ello, todas estas limitaciones

Tabla 3. Diferencias entre ambas soluciones concentradas al 6\% y 12\% de CHO con la situación placebo en todas las variables controladas.

\begin{tabular}{lrccccc}
\hline & Minutos & $\begin{array}{c}\text { Frecuencia } \\
\text { cardiaca }\end{array}$ & Glucosa & Lactato & $\begin{array}{c}\text { Esfuerzo } \\
\text { percibido }\end{array}$ & $\begin{array}{c}\text { Distancia } \\
\text { recorrida }\end{array}$ \\
\hline \multirow{2}{*}{ CHO 6\% } & $\mathbf{0}$ & -4.67 & +0.50 & -0.20 & 0.00 & 0.00 \\
& $\mathbf{2 0}$ & -10.00 & +0.64 & -1.67 & +0.33 & +0.20 \\
& $\mathbf{4 5}$ & -2.00 & +0.03 & -1.80 & -0.33 & +0.14 \\
CHO 12\% & $\mathbf{0}$ & -9.00 & +0.87 & -0.03 & 0.00 & -0.00 \\
& $\mathbf{2 0}$ & -11.00 & -0.13 & -0.57 & -0.34 & -0.12 \\
\hline
\end{tabular}


pudieron influir en la media de la variable de ritmo cardiaco que no fue muy alta comparada con el máximo que debería alcanzarse de acuerdo a esta intensidad.

Probablemente, estas consideraciones podrían haberse solventado en parte con un entrenamiento previo de los participantes sobre la ejecución de la prueba.

\section{5.- CONCLUSIONES.}

No hay evidencias claras ni significativas entre las distintas concentraciones de las soluciones de CHO y el rendimiento deportivo en ninguna de las variables analizadas. Las características individuales y los objetivos del rendimiento deportivo son muy importantes para determinar la concentración de los CHO en la solución utilizada. Los resultados muestran que podría ser más interesante una concentración baja de $\mathrm{CHO}$ al $6 \%$ que una más alta al $12 \%$ de CHO.

Los primeros diez minutos de la prueba pueden verse influenciados positivamente relacionados con una alimentación de baja concentración de $\mathrm{CHO}$ en el ritmo cardiaco, los niveles de glucosa, los niveles de lactato y el esfuerzo percibido de los participantes. Se corroboraron los datos obtenidos por McFarlin, Flynn y Hampton (2007) de que los efectos de la concentración de los niveles de glucosa entre la ingesta de $\mathrm{CHO}$ y placebo hace que no se alcance una hipoglucemia en plasma. Sin embargo, no puede demostrarse que, según Coyle (1999), además de beber agua durante el ejercicio prolongado, la ingesta de $\mathrm{CHO}$ beneficie a los atletas de resistencia para mantener la oxidación de glucosa en un intento de preservar substrato energético (por ejemplo, CHO) necesario para el ejercicio intenso.

Las futuras líneas de investigación podrían requerir en relación a este estudio un entrenamiento previo de los participantes y una estrategia en la concentración del deportista para realizar la máxima intensidad en la prueba.

\section{6.- REFERENCIAS BIBLIOGRÁFICAS.}

Bentleya, D. J., Cox, G. R., Green, D. \& Laursen, P. B. (2007). Maximising performance in triathlon: Applied physiological and nutritional aspects of elite and non-elite competitions. Journal of Science and Medicine in Sport, Artículo en prensa (doi:10.1016/j.jsams.2007.07.010), 1-10.

Bird, S. P., Tarpenning, K. M. \& Marino, F. E. (2006). Effects of liquid carbohydrate/essential amino acid ingestion on acute hormonal response during a single bout of resistance exercise in untrained men. Nutrition (22), 367-375.

Borg, G.A. (1982). Psychophysical bases of perceived exertion, Medicine \& Science in Sports \& Exercise, 14 (5), 377-381.

Bosch, A. N. \& Noakes T. D. (2005). Carbohydrate ingestion during exercise and endurance performance (Invited commentary). Indian Journal of Medicine Research, 121 (5), 634-638.

Bovee, S. E., Silliman, K., Liotta, D. \& Azevedo, J. (2003). Effects of a high fat, high carbohydrate and high protein diet on endurance performance in trained cyclists and untrained controls. Journal of the American Dietetic Association, (pág. 1). A73.

Braham, R., Dawson, B. \& Goodman, C. (2003). The effect of glucosamine supplementation on people experiencing regular knee pain. British Journal of Sports Medicine , 37, 45-49.

Coyle, E. (1999). Physiological determinants of endurance exercise performance. Journal of Science and Medicine in Sport , 3 (2), 181-189.

El Elj, N., Lacb, G., Zaouali, M., Tabka, Z., Gharbi, N. \& El Fazaa, S. (2007). Interaction of high-fat versus high-carbohydrate diets and physical training on energy metabolism in the rat. Science y Sports , 22, 286-288.

Goedecke, J. H., Christie, C., Wilson, G., Dennis, S. C., Noakes, T. D., Hopkins, W. G. et al. (1999). Metabolic Adaptations to a High-Fat Diet in Endurance Cyclists. Metabolism , 48 (12), 15091517.

Haman, F., Péronnet, F., Kenny, J. P., Doucet, E., Massicotte, D., Lavoie, C., et al. (2004). Effects of carbohydrate availability on sustained shivering I. Oxidation of plasma glucose, muscle glycogen, and proteins. Journal of Applied Physiology , 96, 32-40.

McFarlin, B. K., Flynn, M. G. \& Hampton, T. (2007). Carbohydrate consumption during cycling increases in vitro NK cell responses to IL-2 and IFN-c. Brain, Behavior, and Immunity , 21, 202208.

Nassis G.P., Williams C. \& Chisnall P. (1998). Effect of a carbohydrate-electrolyte drink on endurance capacity during prolonged intermittent high intensity running. British Journal of Sports Medicine; 32, 248-252.

Petrie, H. J., Stover, E. A. \& Horswill, C. A. (2004). Nutritional Concerns for the Child and Adolescent Competitor. Nutrition , 20, 620-631.

Powers, S. K. \& Howley, E. T. (2001). Exercise Physiology: Theory and Application. International Edition: McGraw-Hill Higher Education. 
Shirreffs, S. M. (1998). Effects of ingestion of Carbohydrate-electrolyte solutions on exercise performance. International Journal of Sports Medicine , 19, S117-S120.

Vermorel, V., Bitar, A., Vernet, J., Verdier, E. \& Coudert, J. (2003). The extent to which breakfast covers the morning energy expenditure of adolescents with varying levels of physical activity. European Journal of Clinical Nutrition , 57, 310-315.
Vitasovic, R. \& Saldanha, M. (2003). Does medium chain triglyceride play an ergogenic role in endurance exercise performance? Revista Brasileña de Medicina del Deporte , 9 (3), 162168.

Williams, C. (2004). Carbohydrate intake and recovery from exercise. Science y Sports 19, 239-244.

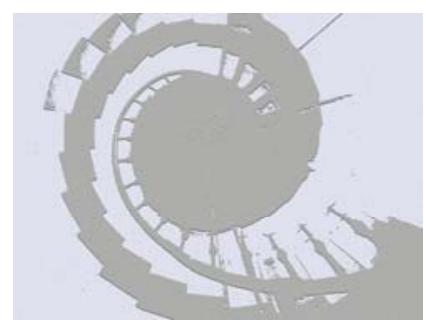

